\title{
ON RECURRENCE OF A RANDOM WALK IN THE PLANE
}

\author{
KAI LAI CHUNG AND TORGNY LINDVALL
}

\begin{abstract}
The purpose of this note is to establish a sufficient condition for recurrence of a random walk $\left(S_{n}\right)$ in $R^{2}$. It follows from it that if $S_{n} / n^{1 / 2}$ is asymptotically normal then we have recurrence.
\end{abstract}

Let $X_{1}, X_{2}, \ldots$ be independent, identically distributed random variables in $R^{k}, k>1$, with common distribution $F$, and let for $n>1, S_{n}=\sum_{1}^{n} X_{i}, S_{0}=0$. The random walk $S=\left(S_{n}\right)_{0}^{\infty}$ has a point of recurrence at $x$ if, for every $\varepsilon>0$,

$$
P\left(\left|S_{n}-x\right|<\varepsilon \text { i.o. }\right)=1 \text {. }
$$

It is well known that the set of recurrence points is either empty or equals the smallest closed additive group containing the support of $F$, see [1] or [3, §8.3]. In the latter case we say that $S$ is recurrent. Also well known is the following criterion: $S$ is recurrent if and only if

$$
\sum_{0}^{\infty} P\left(\left|S_{n}\right| \leqslant \varepsilon\right)=\infty
$$

for some $\varepsilon>0$, see the references above, and (2) holds if and only if

$$
\varlimsup_{r>1} \int_{A} \operatorname{Re} \frac{1}{1-r f(t)} d t=\infty
$$

for all neighborhoods $A$ of 0 , where $f$ is the characteristic function of $F$ : the criterion (3) is Theorem 3 in [1].

The study of recurrence has been carried out, to a large extent, by using (3) and related criteria. For example, in one dimension $E\left[X_{i}\right]=0$ implies recurrence, which is rather easily deduced from (3). In [2] a probabilistic (combinatorial) proof is given that the weaker assumption $S_{n} / n \stackrel{P}{\rightarrow} 0$ is sufficient for recurrence. In that paper it is also claimed that if, in $R^{2}, S_{n} / n^{1 / 2}$ is asymptotically normal, which is the case when $E\left[X_{i}\right]=0$ (zero vector) and $E\left[\left|X_{i}\right|^{2}\right]<\infty$, then we have recurrence. However, the argument indicated for this result in [2], and also in [3, Problem 14, p. 274], is misleading to say the least. This was discovered by students in Chung's class in 1977 and was first corrected by him then: it is the main purpose of this note to settle this matter.

For $y=\left(y_{1}, \ldots, y_{k}\right) \in R^{k}, k \geqslant 2$, we let $|y|=\max _{1<i<k}\left|y_{i}\right|$ throughout.

Received by the editors February 23, 1979.

AMS (MOS) subject classifications (1970). Primary $60 \mathrm{~J} 15$.

(C) 1980 American Mathematical Society 0002-9939/80/0000-0080/\$01.75 
Proposition. Consider a random walk $S=\left(S_{n}\right)_{0}^{\infty}$ in $R^{2}$. If there exists an increasing, continuous function $h \geqslant 0$ on some interval $[0, c]$ such that $\int_{0}^{c}(h(u) / u) d u$ $=\infty$ and

$$
\lim _{n \rightarrow \infty} P\left(\left|S_{n}\right| \leqslant x \cdot n^{1 / 2}\right) \geqslant x^{2} \cdot h(x)
$$

for each $x \in[0, c]$, then $S$ is recurrent.

Proof. Since $x^{2} \cdot h(x)$ is uniformly continuous on $[0, c]$, and since the functions $\inf _{n>k} P\left(\left|S_{n}\right| \leqslant x \cdot n^{1 / 2}\right)$ increase with $x$, the inequality in (4) holds uniformly in $x$ in the sense that for each $\varepsilon>0$ there exist $n(\varepsilon)$ such that

$$
\inf _{n>n(\varepsilon)} P\left(\left|S_{n}\right| \leqslant x \cdot n^{1 / 2}\right) \geqslant x^{2} \cdot h(x)-\varepsilon
$$

for all $x \in[0, c]$. Furthermore, for integers $m \geqslant 1$ we have

$$
\sum_{0}^{\infty} P\left(\left|S_{n}\right| \leqslant m\right) \leqslant 4 m^{2} \cdot \sum_{0}^{\infty} P\left(\left|S_{n}\right| \leqslant 1\right) .
$$

This inequality was proved in [3, Lemma 1, p. 268] for $R^{1}$ with the constant $2 m$ on the right side. The same argument yields the result for $R^{2}$ with the constant $(2 m)^{2}$ due to our definition of $S_{n}$ indicated above. By virtue of (2), it is hence sufficient to prove

$$
\underset{m \rightarrow \infty}{\lim _{m}} m^{-2} \sum_{0}^{\infty} P\left(\left|S_{n}\right| \leqslant m\right)=\infty .
$$

Fix $C>0$ arbitrarily large. Take $B>0$ so large that

$$
\int_{B^{-1}}^{c} \frac{h(u)}{u} d u \geqslant C
$$

Let $\varepsilon>0$ be so small that

$$
\inf _{n>n(\varepsilon)} P\left(\left|S_{n}\right| \leqslant x \cdot n^{1 / 2}\right) \geqslant x^{2} \cdot h(x) / 2
$$

for $B^{-1}<x \leqslant c$, which is possible because of (5) and the monotonicity of $x^{2} \cdot h(x)$. Now, if $n \geqslant n(\varepsilon)$ and $B^{-1} \leqslant m \cdot n^{-1 / 2} \leqslant c$, we have $P\left(\left|S_{n}\right|<m\right) \geqslant m^{2}$. $n^{-1} \cdot h\left(m \cdot n^{-1 / 2}\right) / 2$, so

$$
\begin{aligned}
& \lim _{m \rightarrow \infty} m^{-2} \sum_{0}^{\infty} P\left(\left|S_{n}\right| \leqslant m\right) \geqslant \varliminf_{m \rightarrow \infty} m^{-2} \cdot \sum_{\substack{n>n(\varepsilon) \\
B^{-1}<m \cdot n^{-1 / 2}<c}} P\left(\left|S_{n}\right| \leqslant m\right) \\
& \geqslant \frac{1}{2} \underset{m \rightarrow \infty}{\lim } \int_{m^{2} \cdot c^{-2}}^{B^{2} m^{2}} \frac{h\left(m \cdot x^{-1 / 2}\right)}{x} d x \\
& =\frac{1}{2} \int_{c^{-2}}^{B^{2}} \frac{h\left(x^{-1 / 2}\right)}{x} d x=\int_{B^{-1}}^{c} \frac{h(u)}{u} d u \geqslant C .
\end{aligned}
$$

Since $C$ is arbitrary, $\Sigma_{0}^{\infty} P\left(\left|S_{n}\right| \leqslant 1\right)=\infty$ and hence $S$ is recurrent.

If $S_{n} / n^{1 / 2}$ is asymptotically normal, the Proposition renders $S$ recurrent: simply let $c=1$ and let $h(x)$ be constant $=\min _{|y|<1} g(y)$, where $g$ is the relevant normal density. 
It may be noticed that for every $\beta<2$ there is a $F$ such that $E\left[\left|X_{i}\right|^{\beta}\right]<\infty$ but $S$ is transient. Namely, let $X_{i}=\left(X_{i}^{\prime}, X_{i}^{\prime \prime}\right)$ where the variables $X_{i}^{\prime}, X_{i}^{\prime \prime}$ are independent, stable and symmetric with index $\alpha, \beta<\alpha<2$ : such a distribution has continuous density and all moments of order $<\alpha$ finite, see [4, Lemma 2, p. 545]. With $S_{n}^{\prime}=\sum_{1}^{n} X_{i}^{\prime}$, we obtain

$$
\begin{aligned}
P\left(\left|S_{n}\right| \leqslant 1\right) & =P\left(\left|S_{n}^{\prime}\right| \leqslant 1\right)^{2}=P\left(\left|S_{n}^{\prime} \cdot n^{-1 / \alpha}\right| \leqslant n^{-1 / \alpha}\right)^{2} \\
& =P\left(\left|X_{i}^{\prime}\right| \leqslant n^{-1 / \alpha}\right)^{2} \leqslant 2 \cdot \gamma \cdot n^{-2 / \alpha},
\end{aligned}
$$

where $\gamma$ is the supremum of the density of $F$ on the interval $[-1,1]$. Hence, $\Sigma_{0}^{\infty} P\left(\left|S_{n}\right|<1\right)$ is finite, $S$ is transient.

With a function $h$ as in the Proposition, if $\lim _{n \rightarrow \infty} P\left(\left|S_{n}\right|<x \cdot n\right)>x \cdot h(x)$ for each $x \in[0, c]$ for a one-dimensional random walk $S$, then $S$ is recurrent. This sufficient condition covers the result by Chung and Ornstein, but also, for example, the case when $F$ is a Cauchy distribution such that $S_{n} / n$ is distributed like $X_{1}:$ then $h(x)=$ a suitable constant will do.

Since $h$ is allowed to tend to 0 as $x \searrow 0$, the question arises whether we can find a distribution with slightly heavier tails than that of the Cauchy distribution so that $P\left(\left|S_{n}\right| \leqslant x \cdot n\right) \rightarrow 0$ for all $x>0$ as $n \rightarrow \infty$, and still have recurrence: it turns out that a symmetric distribution on the integers with $P\left(X_{i}=k\right)=\gamma \cdot \log (1+|k|)$ $\cdot\left(1+k^{2}\right)^{-1}, \gamma$ a normalizing constant, is such a distribution. To prove this, Fourier methods seem inevitable.

In order to illuminate that the condition $\int_{0}^{c}(h(u) / u) d u=\infty$ is crucial, let $h>0$ be increasing and such that $\int_{0}^{c}(h(u) / u) d u<\infty$ for some $c>0$ and suppose that

$$
\varlimsup_{n \rightarrow \infty}\left[\sup _{0<x<c} P\left(\left|S_{n}\right| \leqslant x \cdot n^{1 / 2}\right) / x^{2} \cdot h(x)\right]<\infty
$$

for a random walk $S$ in $R^{2}$. Then $S$ is transient, as is readily verified.

\section{REFERENCES}

1. K. L. Chung and W. H. J. Fuchs, On the distribution of values of sums of random variables, Mem. Amer. Math. Soc. No. 6 (1951), 1-12.

2. K. L. Chung and D. Ornstein, On the recurrence of sums of random variables, Bull. Amer. Math. Soc. 68 (1962), 30-32.

3. K. L. Chung, $A$ course in probability theory, 2nd ed., Academic Press, New York, 1974.

4. W. Feller, An introduction to probability theory and its applications. II, Wiley, New York, 1966.

Department of Mathematics, Stanford University, Stanford, California 94305

Department of MATHematics, University of Göteborg, 41296 Göteborg, SWEden 